\title{
Adaptive Light Modulation for Improved Resolution and Efficiency in All-Optical Pulse-Echo Ultrasound
}

\author{
Erwin J. Alles, Richard J. Colchester and Adrien E. Desjardins
}

\begin{abstract}
In biomedical all-optical pulse-echo ultrasound systems, ultrasound is generated with the photoacoustic effect by illuminating an optically absorbing structure with a temporally modulated light source. Nano-second range laser pulses are typically used, which can yield bandwidths exceeding $100 \mathrm{MHz}$. However, acoustical attenuation within tissue or non-uniformities in the detector or source power spectra result in energy loss at the affected frequencies and in a reduced overall system efficiency.

In this work a laser diode is used to generate linear and nonlinear chirp optical modulations that are extended to microsecond time scales, with bandwidths constrained to the system sensitivity. Compared to those obtained using a 2 ns pulsed laser, pulseecho images of a phantom obtained using linear chirp excitation exhibit similar axial resolution (99 versus $92 \mu \mathrm{m}$, respectively) and signal-to-noise ratios (10.3 versus $9.6 \mathrm{~dB}$ ). In addition, the axial point spread function exhibits lower side lobe levels in the case of chirp modulation.

By using non-linear (time-stretched) chirp excitations, where the non-linearity is computed from measurements of the spectral sensitivity of the system, the power spectrum of the imaging system was flattened and its bandwidth broadened. Consequently, the point spread function has a narrower axial extent and still lower side-lobe levels. Pulse-echo images acquired with time-stretched chirps as optical modulation have higher axial resolution $(64 \mu \mathrm{m})$ than those obtained with linear chirps, at the expense of a lower signal-to-noise ratio $(6.8 \mathrm{~dB})$. Using a linear or time-stretched chirp, the conversion efficiency from optical power to acoustical pressure improved by a factor of 70 or 61 , respectively, compared to that obtained with pulsed excitation.
\end{abstract}

\section{INTRODUCTION}

With all-optical pulse-echo ultrasound, sound is generated optically by irradiating an optically absorbing coating or structure. The absorbed optical energy gives rise to a local pressure increase, which propagates through the surrounding medium as an acoustic wave [1]. Reflections from within the medium are received with optical ultrasound sensors, and the corresponding signals are processed to generated images. All-optical pulse-echo ultrasound imaging [2-11] is very promising for minimally invasive clinical procedures; high sensitivity can be achieved with MRI-compatible sources and receivers that are fabricated at the distal end of optical fibres. In addition,

E. Alles, R. Colchester and A. Desjardins are with the Department of Medical Physics \& Biomedical Engineering, University College London, Malet Place Engineering Building, London WC1E 6BT, United Kingdom. Corresponding author: E. Alles, E.Alles@UCL.ac.uk.

The authors wish to thank Piotr Kostecki for technical assistance with the laser diode, and James Guggenheim for assistance with the planar Fabry-Pérot measurements. This research was funded by the European Research Council through European Starting Grant 310970 MOPHIM. sufficiently high pressure levels can be achieved to allow for therapeutic high-intensity focussed ultrasound $[12,13]$.

Conventionally, nanosecond-scale light pulses are used to obtain high acoustic pressures and bandwidths in excess of $100 \mathrm{MHz}$ [4, 14]. However, the light sources tend to be bulky and expensive, and in addition they typically offer no control over the pulse shape. Consequently, the acoustic power spectrum tends to be fixed, whereas different applications require different resolutions and penetration depths. Especially at large penetration depths, high frequencies are attenuated within the tissue, resulting in a narrower received bandwidth. Moreover, the optical absorbers typically employed in fibre-optic ultrasound sources can exhibit strong acoustical attenuation, and hence much narrower transmit bandwidths are often observed $[2,15,16]$. As a result, a significant portion of the optical energy is contained outside the bandwidth of the generated acoustical signal, and hence effectively lost.

To maximise the conversion efficiency from optical to acoustical energy, the optical energy should be constrained both to the bandwidth supported by the optically absorbing structure, and to the bandwidth required by the application. This bandwidth limitation could be achieved by employing modulated CW lasers to perform frequency domain imaging [17, 18]. While in this case spectral matching is trivial, this method requires multiple measurements at different modulation frequencies to reconstruct a single time-domain image.

In this study, a low-cost, portable laser diode was used in conjunction with a high-bandwidth driver (combined dimensions: $65 \mathrm{~mm} \times 85 \mathrm{~mm} \times 20 \mathrm{~mm}$, total cost: $£ 3500 \mathrm{GBP})$, which enabled variation of the shape of the power spectrum of the optical modulation. As the peak power of these laser diodes is typically five orders of magnitude lower than that of typically employed pulsed light sources [16], coded excitation was used in conjunction with pulse compression to recover the B-mode image signal-to-noise ratio (SNR) [19, Ch.3]. Here, linear chirps with flat bandwidths [20] were used, of which the pulse energy is determined by the pulse duration. Linear chirps have previously been used as optical modulation to improve the SNR of photoacoustic images [21-23], but to our knowledge they have not been applied to improve the efficiency of alloptical pulse-echo ultrasound imaging systems.

The power spectrum of the optical modulation can be modified to compensate for non-idealities in the power spectrum caused by the transduction technology, such as non-uniform detector sensitivity or non-uniform acoustic power. While this type of spectral shaping can be performed using amplitude modulation, it would significantly decrease the overall acoustical power due to the low maximum optical intensity of a laser diode. Instead, spectral shaping is achieved by varying the 
rate of frequency change with time, thereby effectively timestretching a linear chirp. One class of time-stretched chirps was shown to improve the image quality in photoacoustic imaging [22], but in that work the frequency was restricted to vary with time in a polynomial fashion. In this study, that restriction is lifted and the rate of frequency change is computed on a per-frequency basis to allow for greater flexibility in spectral shaping.

In this work, all-optical pulse-echo imaging is demonstrated using a modulated laser diode and an all-optical sourcereceiver transducer pair designed for biomedical interventional use. While both the source and receiver are known to have non-uniform power spectra $[16,24]$, they provide an effective demonstration of how an optical-to-acoustic transduction efficiency gain can be achieved by limiting the bandwidth of the optical modulation, and how the power spectrum uniformity of the received acoustical signal can be improved through spectral shaping. These demonstrations are achieved through measurements of the axial point spread functions using pulsed, linear chirp, and time-stretched chirp modulations. In addition, Bmode pulse-echo ultrasound images of phantoms are presented, which demonstrate the gain in spatial resolution achieved by applying a time-stretched chirp modulation.

\section{METHODS}

\section{A. Experimental setup}

A step-index optical fibre with a core/cladding diameter of $200 / 220 \mu \mathrm{m}$ was used as ultrasound source. This fibre was dip-coated using the process described in [16], resulting in an optically absorbing coating (transmittance $<5 \%$ ) with a maximum thickness of approximately $30 \mu \mathrm{m}$, consisting of a composite of functionalised carbon nanotubes (CNTs) and polydimethylsiloxane (PDMS). This coating was illuminated using either a pulsed laser (SPOT-10-500-1064, Elforlight, UK) or a modulated laser diode (LU1064M450, Lumics, Germany) driven by a high-frequency driver (BFS-VRM-03HP, Picolas, Germany). Both lasers operated at a wavelength of $1064 \mathrm{~nm}$. The excitation voltage provided to the laser driver was generated by an arbitrary waveform generator (33611A, Agilent, CA, USA) and sampled at $200 \mathrm{MSa} / \mathrm{s}$. One percent of the optical power was tapped using a $99: 1$ fibre optic coupler (FC1064-99B-APC, Thorlabs, Germany) and its modulated intensity was monitored with a fast photodiode (DET10A, Thorlabs, Germany); the remaining $99 \%$ was used to generate the ultrasound. As the light generated by both sources was coupled into the same multi-mode source fibre, the acoustic source geometry was independent of the light source and hence so was the directivity pattern of the acoustic source.

Acoustical detection was performed using either a custom planar Fabry-Pérot etalon (10 $\mu \mathrm{m}$ spacer thickness) featuring a uniform sensitivity [25], or a custom fibre-optic hydrophone with a plano-convex Fabry-Pérot cavity at the distal end, similar in construction to that presented in [26]. While the fibreoptic hydrophone is known to have a non-uniform frequency response [24], it was selected for its high sensitivity and small lateral dimensions $(<250 \mu \mathrm{m})$ suitable for biomedical interventional application. Both sensors were interrogated by

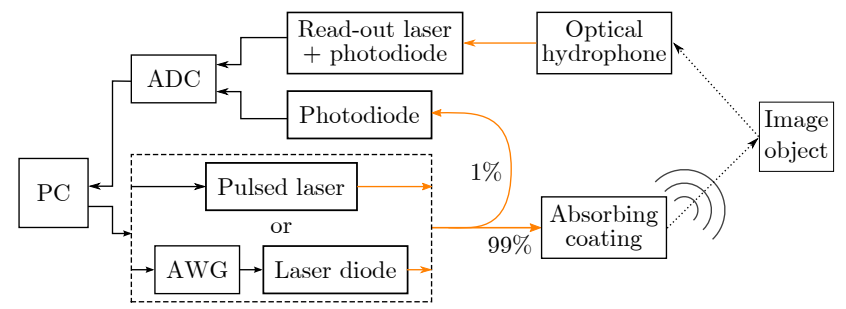

Fig. 1. A schematic of the experimental setup used in this work. Ultrasound is generated by illuminating an optically absorbing coating using either a pulsed laser or a modulated laser diode. One percent of the emitted light is tapped from the output to be recorded by a photodiode. The transmitted ultrasound scatters off the image object and is recorded using an optical hydrophone. A desktop PC was used for data acquisition and synchronisation and to program the arbitrary waveform generator (AWG)

measuring the sensor's reflectivity using a tunable laser (TUNICS T100S-HP, Yenista, France) and a custom photodiode. The wavelength of the interrogation laser was continuously adjusted to correspond with the peak derivative of the hydrophone's cavity transfer function [25].

To measure the acoustic power spectrum of the optically absorbing coating, acoustic transmission measurements were performed at a distance of $2 \mathrm{~mm}$ with the coated source fibre centred and at normal incidence to the planar sensor. Pulseecho measurements were acquired with the coated source fibre and optical fibre hydrophone positioned adjacent to each other and facing the same direction. The signals from the intensity monitoring and hydrophone interrogation photodiodes were recorded at $100 \mathrm{MSa} / \mathrm{s}$ using a digitiser card (NI PCI-5142, National Instruments, TX, USA). All equipment was controlled by a desktop PC running MATLAB R2012b and LabVIEW 2012 SP1. The setup is shown schematically in Fig. 1.

\section{B. Light modulation}

The light generated by the laser diode was modulated by a chirp of which the frequency increased monotonically with time. Two types of chirp modulations were considered: a linear chirp and a time-stretched version of the linear chirp. Both chirp signals $c(t)$ had the general form

$$
c(t)=A \sin \left(\phi_{0}+2 \pi \int_{0}^{t} f(\tau) d \tau\right),
$$

where $f(t)$ is the time-dependent frequency, the integration runs over time $\tau, \phi_{0}$ is a constant phase offset, and $A$ is the amplitude of the chirp signal. In the case of a linear chirp, the rate of frequency change with time $\dot{f}(t)$ is constant, i.e.,

$$
\begin{aligned}
\dot{f}(t) & =\frac{f_{\max }-f_{\min }}{\Delta}=\text { constant }, \\
\Rightarrow f(t) & =f_{\min }+\frac{f_{\max }-f_{\min }}{\Delta} t,
\end{aligned}
$$

where $f_{\min }, f_{\max }$, and $\Delta$ are the minimum frequency, maximum frequency, and chirp duration, respectively, and the integration constant is determined by requiring $f(0)=f_{\text {min }}$. 
Consequently, the analytic expression for a linear chirp is given by

$$
\begin{aligned}
c(t) & =A \sin \left(\phi_{0}+2 \pi \int_{0}^{t} \int_{0}^{\tau} \dot{f}(\theta) d \theta d \tau\right) \\
& =A \sin \left(\phi_{0}+2 \pi\left[f_{\min } t+\frac{f_{\max }-f_{\min }}{2 \Delta} t^{2}\right]\right) .
\end{aligned}
$$

The idealised case, in which the power spectrum $|P(\omega)|^{2}$ of the acoustical response $p(t)$ is uniform across its bandwidth, i.e.,

$$
|P(\omega)|^{2}=|\mathcal{F}\{p(t)\}|^{2}=\text { constant, } \quad f_{\min } \leq\left|\frac{\omega}{2 \pi}\right| \leq f_{\max },
$$

where $\mathcal{F}\{$.$\} indicates the Fourier transform and \omega=2 \pi f$ is the angular frequency, yields the smallest possible axial point spread function within the bandwidth constraints. However, in reality there are non-uniformities in the power spectrum that decrease the spatial resolution of the imaging system. To correct for these non-uniformities, a time-stretched chirp signal can be used, of which the rate of frequency change $\dot{f}(t)$ is adjusted so that more time is spent at frequencies corresponding to low acoustic power. To compensate for the non-uniformities in the power spectrum, the rate of frequency change is set to

$$
\dot{f}\left(\omega=2 \pi\left[f_{\min }+\frac{f_{\max }-f_{\min }}{\Delta} t\right]\right) \propto|P(\omega)|^{2},
$$

where use was made of equation (3) to convert frequency into time. On a discrete time grid, equation (6) yields $\dot{f}(t)$ on a non-uniform time axis, which was converted to a uniform axis by means of nearest-neighbour interpolation and then scaled to yield the correct duration $\Delta$. Finally, the non-linear, or timestretched, chirp signal was obtained using equation (4) where the integrations were evaluated numerically.

For a fixed temporal step size (i.e., for a fixed sample rate), the fidelity with which non-uniformities in the power spectrum can be compensated increases with the chirp duration, as the chirp duration is inversely related to the rate of frequency change $\dot{f}(t)$. Consequently, for a longer chirp duration the rate of frequency change can be sampled more densely and hence the numerical integrations in equation (4) can be evaluated more accurately. To demonstrate this, results for linear and time-stretched chirp lengths of both 4 and $40 \mu$ s are provided.

However, in a pulse-echo imaging context, a longer pulse duration could result in motion artifacts and reduced image quality. Additionally, longer pulse durations result in longer A-scan acquisition times and consequently lower image frame rates. The linear chirps used ranged between 0 and $50 \mathrm{MHz}$, corresponding to the full bandwidth of the digitiser card, and the acoustical response to this optical modulation was used as input $p(t)$ to compute the time-stretched chirp. The timestretched chirp was computed for frequencies between 3 and $35 \mathrm{MHz}$, corresponding to the bandwidth where the received ultrasound signal was above the noise level.

The chirped input signals were provided to the laser diode driver by the arbitrary wave generator in the form of a discretised voltage time series. The analogue voltage values were scaled and offset so that they ranged between the extremum values of $0.09 \mathrm{~V}$ and $0.7 \mathrm{~V}$, corresponding to zero and maximum light intensity, respectively. For all three optical modulations, the repetition rate of the chirps was chosen such that the time-averaged optical power of excitation light delivered to the absorbing coating was $O=5.00 \mathrm{~mW}$. At this optical power level, reproducible acoustical responses were generated without causing damage to the coating. For the pulsed laser, this amounted to a repetition rate of $175 \mathrm{~Hz}$, whereas the scaled chirp signals were zero-padded to obtain a repetition rate of $3350 \mathrm{~Hz}$. As the discontinuities introduced by this zero-padding give rise to additional unwanted acoustical responses, the offset was tapered using a Tukey window with parameter $\alpha=11 \%$ (determined empirically). All acoustical responses presented below are averages over the relevant number of acquisitions (175 for pulses or 3350 for chirps) to ensure that the same total optical energy was associated with each measurement.

\section{Signal processing and analysis}

Power spectra of both the optical modulation and the acoustical response were computed using MATLAB's FFT routine. Bandwidths were defined by the $-6 \mathrm{~dB}$ level in $|P(\omega)|^{2}$ relative to the maximum power. Pulse compression based on crosscorrelations using matched filters was applied to determine the axial point spread functions (PSFs), where the filters were obtained by time-windowing the A-scan measurements of echoes off a steel block. Main lobe widths were determined by locating (by means of cubic interpolation) the axial positions where the PSF was reduced to half its maximum value. Side-lobe levels were defined as the mean of the maximum amplitudes of the first side-lobe occurring before and after the main lobe. Envelopes of PSFs and images were computed using the absolute value of MATLAB's Hilbert transform.

A measure of the conversion efficiency at which optical power is converted to acoustical pressure, $\eta$, was obtained by computing the ratio between the maximum amplitude of the acoustical measurement (averaged over either 175 or 3350 acquisitions to suppress noise) and the optical power during the pulse or chirp,

$$
\eta=\frac{\max _{t}|p(t)|}{O /(R \cdot \Delta)}
$$

where $\max _{t}$ is the maximum value of its argument, $O$ is the time-averaged optical power, $R$ is the relevant repetition rate and $\Delta$ the optical pulse duration. As the applied hydrophone is not calibrated, this efficiency provides a relative measure in arbitrary units. The conversion efficiency ratio $\epsilon$ is defined as

$$
\epsilon=\frac{\eta}{\eta_{\text {pulsed }}}
$$

where $\eta_{\text {pulsed }}$ is the conversion efficiency obtained with pulsed excitation.

\section{Phantom imaging}

Two separate phantoms were used in this study. The first phantom consisted of a steel block placed, at right angles, 
at a distance of $4 \mathrm{~mm}$ from the source/receiver fibre pair. As these fibres were facing the same direction, pulse-echo data were acquired to obtain the matched filters applied during pulse compression and to measure the axial point spread function.

The second phantom consisted of two layers of parallel carbon fibres $(100 \mu \mathrm{m}$ diameter $)$ spaced $1 \mathrm{~mm}$ apart. The first layer was located at a distance of approximately $4 \mathrm{~mm}$ from the fibres, the second layer at roughly $8 \mathrm{~mm}$. A twodimensional pulse-echo image of this second phantom was acquired by translating the source and receive fibres along a straight path (forming the lateral direction) perpendicular to the fibre orientation. This translation was performed in an automated fashion using a motorised stage (MTS50/M-Z8 + TDC001, Thorlabs, Germany), and acoustical pulse-echo Ascans were acquired at $50 \mu \mathrm{m}$ intervals. A-scans averaged over the same numbers of acquisitions as above were recorded, and pulse compression based on matched filtering was applied to each A-scan.

Image reconstruction was performed using the $\mathrm{k}$-Wave toolbox [27], and the envelope of the resulting B-mode images was taken along the axial dimension. In the case of the timestretched chirp, A-scans were first band-pass filtered with a pass-band between $3 \leq f \leq 35 \mathrm{MHz}$, tapered with a Tukey window (parameter $\alpha=1.5 \%$ ), to suppress signals at frequencies that were not excited. Signal-to-noise ratios (SNRs) were computed as the ratio between the mean image amplitude within a region of interest containing the image of a single carbon fibre over that of an equally large region containing only noise.

\section{RESULTS}

\section{A. Pulsed optical excitation}

The large bandwidth contained in the $2 \mathrm{~ns}$ long optical pulse is visible in Fig. 2 (thin line, bottom panel). Using the planar sensor exhibiting a uniform sensitivity, the acoustic field generated through pulsed excitation was found to have a $-6 \mathrm{dBb}$ bandwidth ranging from $2.6 \leq f \leq 25.8 \mathrm{MHz}$ (thick dotted line, bottom panel), which is significantly lower than the theoretical bandwidth. In addition, the fibre hydrophone was observed to have a non-uniform sensitivity, resulting in a bandwidth of $46 \%$ around a peak power frequency of $8.1 \mathrm{MHz}$ (thick solid line, bottom panel) and a finite temporal extent of the acoustical response of about half a microsecond (thick solid line, top panel).

\section{B. Pulsed versus linear chirp excitation}

When a $4 \mu$ s linear chirp with a frequency ranging between 0 and $50 \mathrm{Mhz}$ is used as optical excitation (Fig. 3, thin dashed line, top panel), the acoustical response (thick dashed line, top panel) exhibits the same non-uniformities in the acoustical emission spectrum and detection sensitivity through strong amplitude modulation. As the power spectrum of the optical chirp signal (thin dashed line, bottom panel) is virtually identical to that of the pulsed excitation, the power spectrum of the acoustical response to optical chirped excitation (thick dashed line, bottom panel) is very similar to that for pulsed
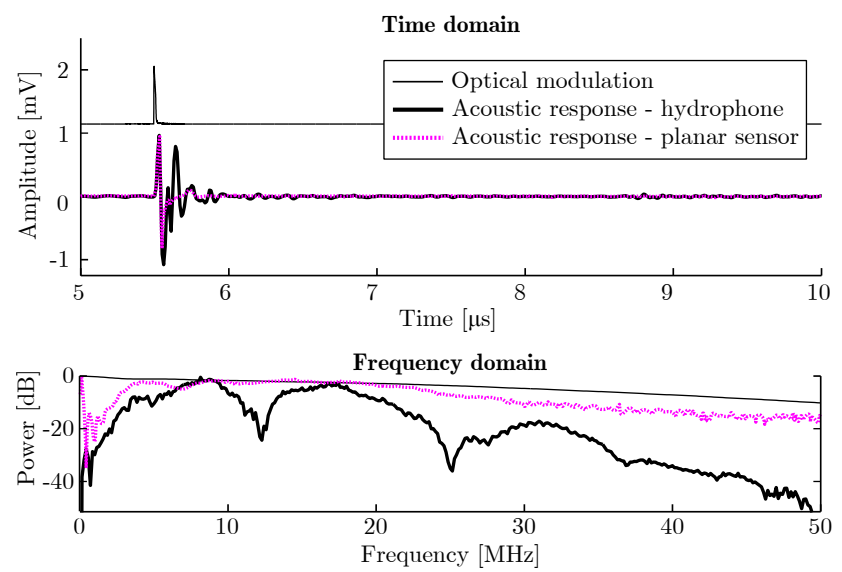

Fig. 2. Acoustical measurements using a 2 ns duration optical pulse. In the top panel, the optical pulse (thin line) is shown, together with the acoustical signal measured with both the fibre hydrophone (thick solid line) and the planar sensor (thick dotted line). The bottom panel shows the corresponding power spectra. To improve visibility and facilitate qualitative comparison, the acoustical responses have been normalised to a maximum amplitude of $+1 \mathrm{mV}$ (actual extreme values: $-323 \mathrm{mV}$ and $290 \mathrm{mV}$ ), the optical modulation was acquired using a $-40 \mathrm{~dB}$ neutral density filter to avoid saturation of the photodiode, an offset of $1.15 \mathrm{mV}$ was added to the optical modulation trace, and the optical modulation traces were temporally delayed to ensure the optical and acoustical signals all start at $t=5.5 \mu$ s. The power spectra were normalised to $0 \mathrm{~dB}$.

excitation between 3 and $35 \mathrm{MHz}$. The differences observed below $3 \mathrm{MHz}$ are due to the Tukey-windowed offset applied to the chirp excitation (visible between $5.25 \leq t \leq 5.5 \mu$ s and $9.5 \leq t \leq 9.75 \mu$ s in the thin dashed line in the top panel), and the differences above $35 \mathrm{MHz}$ are due in part to noise and in part to harmonics generated by the non-linear response of the laser driver.

The apparent amplitude modulation visible at higher frequencies in the chirp optical modulation (dashed thin line) is due to the low sampling rate of the acquisition card (100 MSa/s), and this amplitude modulation disappears (dotted thin line) when the same signal is recorded at $250 \mathrm{MSa} / \mathrm{s}$ using a different acquisition card (M4i.4420-x8, Spectrum, Germany). As the frequency content of the chirp modulation does not exceed the Nyquist bandwidth of the acquisition card, the power spectrum of the chirp optical modulation sampled at $100 \mathrm{MSa} / \mathrm{s}$ does not reflect the time domain amplitude modulation. In addition, the acoustic responses to both pulsed and chirp optical modulation exhibit still narrower bandwidths, and hence a sampling rate of $100 \mathrm{MSa} / \mathrm{s}$ was sufficient for the experiments presented in this work.

\section{Linear versus time-stretched chirp excitation}

The process of time-stretching a linear chirp to compensate for non-uniformities in the acoustical power spectrum is illustrated in Fig. 4. The power spectrum of the acoustical response to a linear chirp for a chirp duration of $4 \mu$ s (bottom panel, dashed line) was used to compute the rate of frequency change $\dot{f}(t)$ for frequencies $3 \leq f \leq 35 \mathrm{MHz}$, corresponding to the 


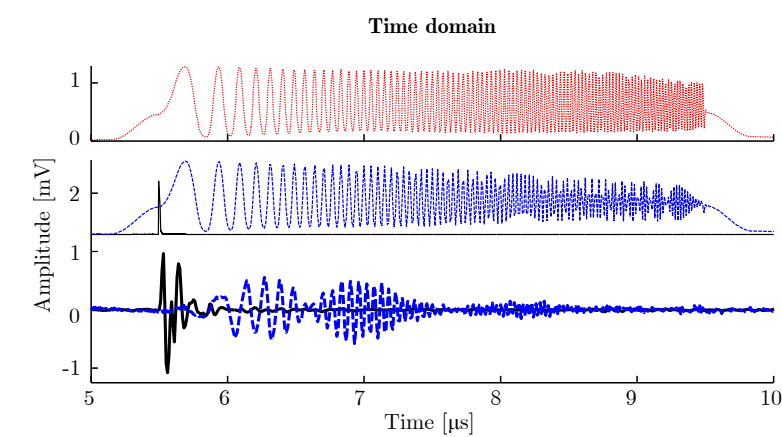

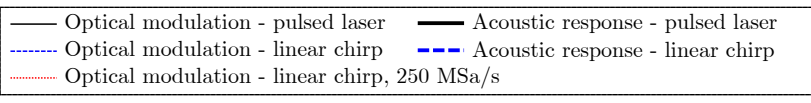

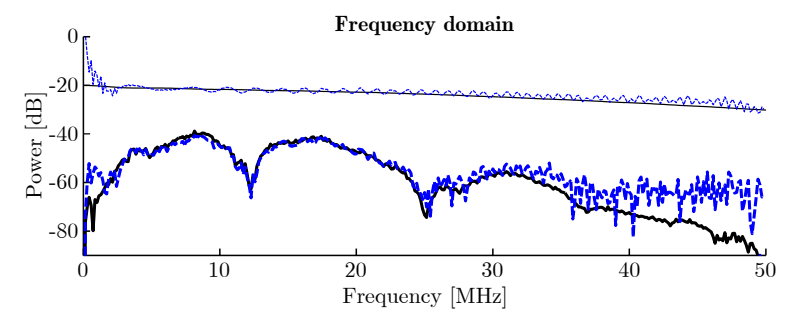

Fig. 3. Comparison between 2 ns duration pulsed or $4 \mu$ s duration linear chirp optical modulation. The data obtained with pulsed modulation is identical to that in Fig. 2. In the top panel, the optical modulation signals (thin lines) and the corresponding acoustical A-scans (thick lines) are shown for a pulsed laser (solid lines) and a laser diode modulated with a linear chirp (dashed lines sampled at $100 \mathrm{MSa} / \mathrm{s}$, dotted line sampled at $250 \mathrm{MSa} / \mathrm{s}$ ). The optical modulation signals are scaled and offset to improve visibility. In the bottom panel, the corresponding power spectra are shown. While the power spectra of both optical modulation signals are smooth and virtually constant, the acoustical responses exhibit strong non-flatness and an overall decrease with increasing frequency. To improve visibility and facilitate qualitative comparison, an offset of $1.15 \mathrm{mV}$ was added to both optical modulation traces sampled at $100 \mathrm{MSa} / \mathrm{s}$, and the optical modulation traces were temporally delayed to ensure the optical and acoustical signals all start at $t=5.5 \mu \mathrm{s}$. The power spectra of the pulsed optical modulation, linear chirp optical modulation, and acoustical response to the pulsed laser are offset by +11 , +20 , and $-46 \mathrm{~dB}$, respectively.

region where the signal is stronger than the noise. Across this bandwidth, the power spectrum of the resulting time-stretched chirp optical modulation ( $4 \mu$ s duration, top panel, dotted line) is proportional to the inverse of the power spectrum of the acoustical response to a linear chirp, and the power spectrum of the acoustical response to this time-stretched chirp (dotted line, bottom panel) is significantly more uniform. Its peak power frequency has increased from 8.1 to $13.7 \mathrm{MHz}$, and the trough around $25 \mathrm{MHz}$ has been removed. However, due to the remaining drop in acoustical power at $12 \mathrm{MHz}$, the $-6 \mathrm{~dB}$ bandwidth has not been improved. When a longer chirp duration of $40 \mu \mathrm{s}$ is used (dash-dotted line), even the sharp drop at $12 \mathrm{MHz}$ is accurately corrected and the resulting power spectrum is nearly uniform. Note that the obtained acoustical power spectrum is more uniform than that of the acoustic pulse generated by the coating (dotted line in Fig 2), demonstrating that the time-stretching mechanism compensates for both the emission and detection spectra simultaneously. The structure visible at $24 \mathrm{MHz}$ is due to interference of harmonics generated

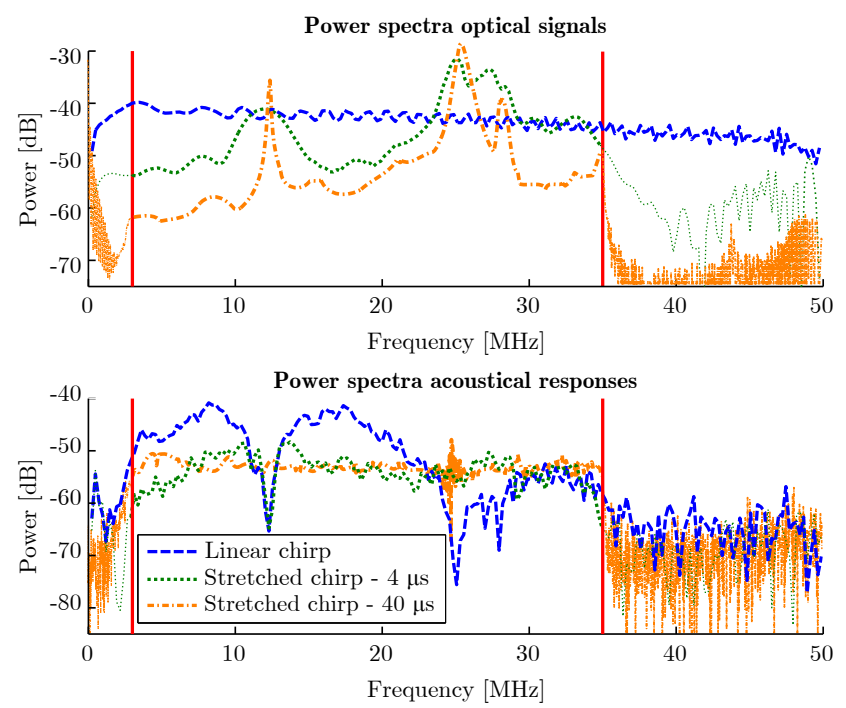

Fig. 4. The effect of time-stretching a linear chirp optical excitation on the acoustical response. In the top panel, the measured power spectra of a linear chirp (dashed line, $4 \mu$ s duration) and a time-stretched chirp (dotted line, $4 \mu \mathrm{s}$ duration) optical modulation are shown. Time-stretching was computed using the acoustical response to a linear chirp optical excitation of Fig. 3, and was applied to flatten the power spectrum of the acoustical response between 3 and $35 \mathrm{MHz}$ (indicated by the solid lines), corresponding to the bandwidth in which the acoustical power was above the noise level. In the bottom panel, the power spectra of the acoustical responses to the linear and time-stretched chirp optical excitation are shown. The response to the linear chirp (dashed line) is identical to that in Fig. 3, whereas the response to the time-stretched chirp (dotted line) has a much flatter spectrum between 3 and $35 \mathrm{MHz}$. The acoustical response to a $40 \mu$ s time-stretched chirp optical modulation has a nearly uniform power spectrum (dash-dotted line).

by the non-linearity of the laser driver.

The energy conversion efficiency ratios for the linear and time-stretched chirps ( $4 \mu$ s duration) were found to be $\epsilon_{\text {linear }}=$ 69.6, and $\epsilon_{\text {stretched }}=60.9$, respectively. The conversion efficiency is hence almost two orders of magnitude higher for the chirp excitations than for pulsed excitation, as in the former cases the optical energy is confined to the transmission and detection bandwidths of the acquisition system.

\section{Axial point spread functions}

The point spread function (PSF) obtained after pulse compression in the case of pulsed excitation (solid line in Fig. 5) exhibits a main lobe width of $68 \mathrm{~ns}$, corresponding to $99 \mu \mathrm{m}$, and a relative side-lobe level of 0.41 . As the acoustical power spectrum and bandwidth obtained using linear chirp excitation are very similar to that obtained using pulsed excitation (see Fig. 3), the main lobe width $(63 \mathrm{~ns} / 92 \mu \mathrm{m})$ and relative side-lobe level (0.37) of the PSF using a linear chirp (dashed line) are comparable. However, when a time-stretched chirp is applied, the main lobe width is smaller (44 ns / $64 \mu \mathrm{m}$, dotted line) due to the broader bandwidth demonstrated in the bottom panel of Fig. 4, and relative side lobe levels are further reduced to 0.30 . The reduction in side lobe levels observed for both chirp excitations was due to the smoothly varying amplitude 


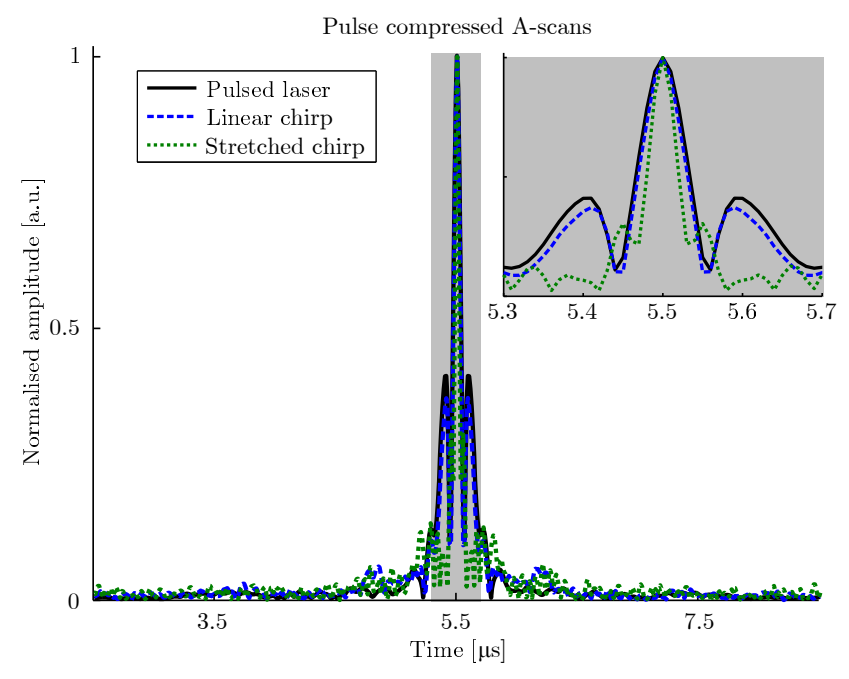

Fig. 5. Normalised envelope of the axial point spread functions of the setup using a pulsed (solid line), linear chirp (dashed line, $4 \mu$ s duration) and timestretched chirp (dotted line, $4 \mu$ s duration) optical modulation. Matched filter pulse compression was applied, and the envelopes of the point spread functions are shown. The inset contains a magnification of the region highlighted in gray.

modulation introduced by the non-uniform detector sensitivity, which suppresses discontinuities such as those observed in the acoustic response to pulsed excitation (Fig. 3). As a result, the acoustic responses to chirp excitations are effectively apodised, which decreases pulse compression side lobe levels [19, Ch.3].

\section{E. Phantom B-mode imaging}

In the reconstructed images, the carbon fibres were clearly visible (Fig. 6). In the top left panel, the image obtained using pulsed optical excitation is shown, which clearly reproduces the structure of the phantom at an SNR of $10.3 \mathrm{~dB}$. The regions of interest used to determine the signal and noise levels are indicated by the solid and dotted boxes, respectively. The phantom geometry, orientation of the source and receive fibres, and scan direction are shown in the top right panel.

A similar image quality is obtained when a linear chirp is used, as is visible in the bottom left panel. The image is virtually identical to that obtained using pulsed excitation, and has a similar SNR of $9.6 \mathrm{~dB}$. When a time-stretched chirp is used, the improved bandwidth results in a higher axial resolution that manifests itself in the higher resolution observed in the bottom right panel. However, as in this case more time is spent at frequencies of low acoustical power or sensitivity, thereby decreasing the mean acoustical power, the $\mathrm{SNR}$ is reduced to $6.8 \mathrm{~dB}$.

To facilitate image quality comparison, the sections indicated by the dashed boxes are magnified in Fig. 7. Normalised profiles through the images of one of the carbon fibres (along the dashed lines) are shown in the top right panel. Due to the lower sidelobes demonstrated in Fig. 5, the profile obtained using a linear chirp (dashed line) is marginally narrower (as is visible at a depth of around $4.4 \mathrm{~mm}$ ) than that obtained using pulsed excitation (solid line). The profile obtained from
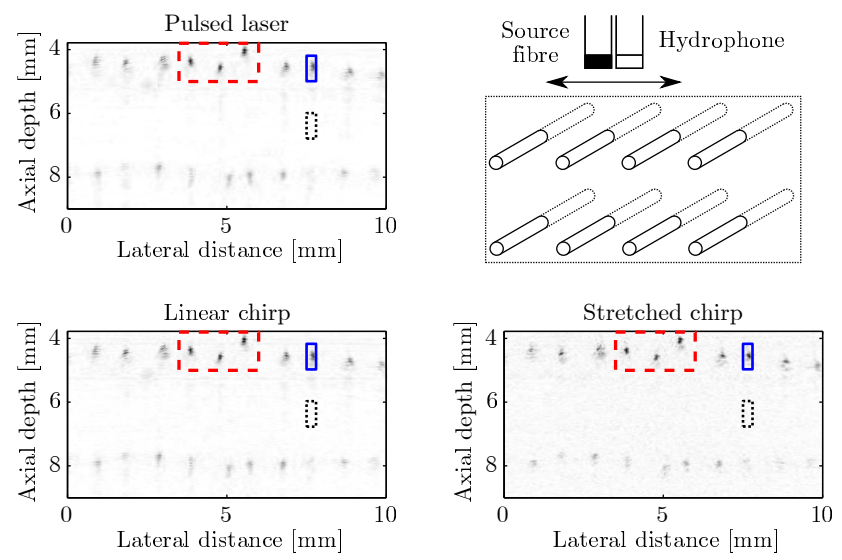

Fig. 6. Images obtained of a phantom (top right) using a pulse (top left, $4 \mu \mathrm{s}$ duration), a linear chirp (bottom left, $4 \mu$ s duration) or a time-stretched chirp (bottom right) as optical modulation. Data were acquired by linearly translating the source and receiver fibre pair and recording A-scans at $50 \mu \mathrm{m}$ intervals, and images were reconstructed from pulse compressed radio-frequency (RF) data. In the time-stretched chirp case, RF data was filtered using a band-pass filter with a pass band between 3 and $35 \mathrm{MHz}$. The regions indicated by the dashed boxes are magnified in Fig. 7, and the regions of interest used to determine the signal and noise levels are indicated by the solid and dotted boxes, respectively.
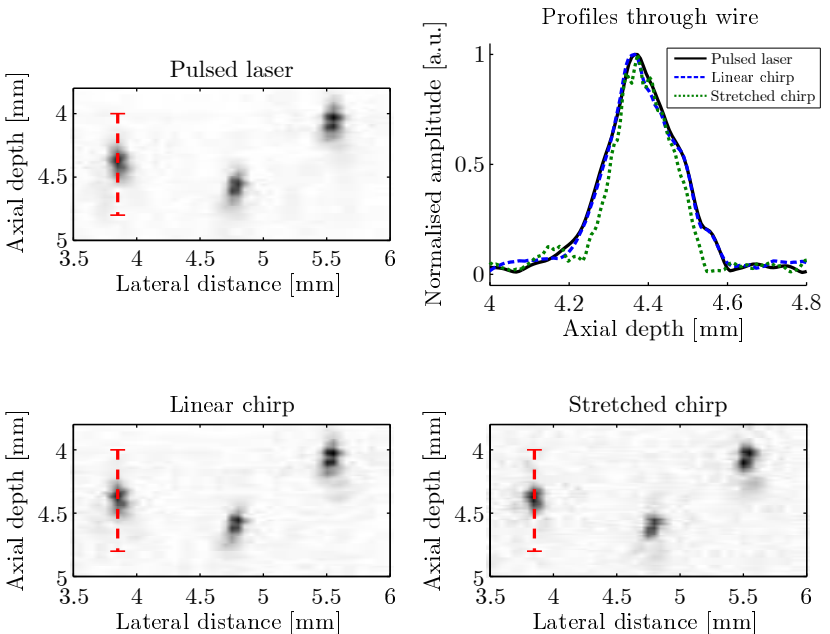

Fig. 7. Magnifications of the dashed boxes in the images in Fig. 6, and amplitude profiles (top right) through the image along the path indicated by the dashed line.

images generated using a time-stretched chirp is narrower still (dotted line) due to the increased bandwidth.

\section{Discussion}

This study provided the first demonstration of applying modulated instead of pulsed excitation to all-optical pulse-echo ultrasound. The presented advantages of using modulated instead of pulsed optical excitation depend strongly on the bandwidths of the applied source and receiver technologies. For sufficiently thin optically absorbing structures, thin-film ultrasound 
generation occurs and the generated acoustic field exhibits a wide bandwidth. However, if a thicker source structure is employed, such as the $30 \mu \mathrm{m}$ thick coating used in this work, bulk ultrasound generation occurs. In this case, the generated acoustic field is convolved with the spatial distribution of the absorbed optical energy, and consequently an acoustic field is transmitted that has a narrower, non-uniform bandwidth.

Optically-absorbing CNT-PDMS nanocomposite coatings were chosen in this study as promising pulse-echo ultrasound imaging results were previously achieved [11, 12, 28], and in addition these coatings tend to be robust and easy to fabricate. However, acoustic attenuation within this type of coating results in lower bandwidths than those previously achieved (cf. [14, 29]); for instance, as demonstrated in [30], a $25 \mu \mathrm{m}$ thick carbon-black PDMS coating gives rise to $15 \mathrm{~dB}$ attenuation at a frequency of $80 \mathrm{MHz}$. For many applications, the resulting source bandwidth decrease is acceptable. For instance, in ultrasound imaging applications requiring penetration depths exceeding a few millimeters, acoustical attenuation within the tissue limits the effective bandwidth to $30 \mathrm{MHz}$ or less. Using bandwidth-limited optical modulation, the system's efficiency can therefore be greatly improved.

In this work, time-stretched chirp optical modulations were successfully used to simultaneously compensate for nonuniformities in both the source power spectrum and detector sensitivity, and the resulting pulse-echo ultrasound image exhibited a higher spatial resolution. Transmitting time-stretched chirps to compensate for a non-uniform detector sensitivity is preferred to applying calibration curves, as the latter method tends to amplify and spectrally colour noise.

The accuracy with which the acoustic power spectrum can be flattened can be improved by extending the duration of the optical modulation. However, in an in vivo imaging setting, using optical excitations with longer durations could result in motion artifacts that suppress the benefits of spectral flattening. Harmonic components in the optical signal generated by the laser driver further limit the spectral flattening accuracy. In addition, due to acoustic diffraction, the pulse compression scheme will be most accurate in the location where the matched filter was obtained.

Even in the ideal case in which the transmitted ultrasound has ultra-high bandwidth (e.g., [14, 29]) and the receiver exhibits uniform, broadband sensitivity (e.g., [24, 31-33]), using modulated optical excitation could prove beneficial. For instance, optical excitations with extended durations could improve the SNR in situations where the peak power is limited by the material tolerances of the optically-absorbing coating or the underlying substrate. For instance, the damage threshold for fused silica $\left(1 \mathrm{GW} / \mathrm{cm}^{2}\right)$ limits the energy contained in a $2 \mathrm{~ns}$ long pulse delivered in a $200 \mu \mathrm{m}$ core optical fibre to approximately $600 \mu \mathrm{J}$ [34]. The optically-absorbing coating is damaged at much lower values, typically around $30 \mu \mathrm{J}$. Using a modulated laser diode, broad-band optical energy can be spread out over longer time periods while keeping peak power levels below material tolerances.

Contrary to piezo-electric ultrasound transducers, optically generated ultrasound does not rely on resonance to generate the acoustical energy. As a consequence, the bandwidth and centre frequency can be optimised for different imaging contexts. Using the same optical acoustic source and modulated light source, ultrasound can be generated with a bandwidth that is either very narrow (useful for Doppler flowmetry or sample characterisation), very broad (for high resolution imaging), or intermediate (for larger penetration depth). This approach can be extended to photoacoustic imaging, where the light is absorbed within biological tissue or other samples. It has been shown that the emitted acoustical spectrum depends on the geometry of the optical absorber [35, 36], and hence by applying modulated instead of pulsed light the spectral sensitivity of the system can be tuned to the specific geometry under study. In a similar manner, an all-optical pulse-echo ultrasound system could be tuned using modulated optical excitation to enhance sensitivity for particular structures such as micro-bubbles.

\section{CONCLUSION}

By switching from pulsed optical excitation to an optical modulation of larger temporal extent, the bandwidth of the optical modulation can be constrained to those frequencies that are generated and detected by the optically absorbing coating and receiver. As a result, losses due to acoustical attenuation within the coating or tissue or due to a non-uniform detection sensitivity can be avoided. With the ultrasound source and receiver used in this study, the conversion efficiency of optical energy to detected acoustic signal was increased by almost two orders of magnitude. Pulse-echo B-mode images can be obtained using a modulated laser diode for transmission, with signal-to-noise ratios similar to those of images acquired using a pulsed laser, despite a peak power that is lower by nearly five orders of magnitude. In addition, by using non-linear (time-stretched) chirp excitations, where the non-linearity is computed from measurements of the spectral sensitivity of the system, the non-uniformities in the emitted acoustical power spectrum and the detector sensitivity can simultaneously be reduced, resulting in an increased bandwidth and consequently in an improved spatial resolution.

\section{REFERENCES}

[1] PC Beard. Biomedical photoacoustic imaging. Interface focus, 1(4):602-631, 2011.

[2] Y Hou, J-S Kim, S Ashkenazi, S-W Huang, LJ Guo, and $\mathrm{M}$ O'Donnell. Broadband all-optical ultrasound transducers. Applied Physics Letters, 91(7):073507, 2007.

[3] S-W Huang, S Ashkenazi, Y Hou, RS Witte, and M O'Donnell. Toward fiber-based high-frequency 3d ultrasound imaging. In Proceedings SPIE BiOS, pages 643728-643728-8. International Society for Optics and Photonics, 2007.

[4] Y Hou, J-S Kim, S-W Huang, S Ashkenazi, L Jay Guo, and M O'Donnell. Characterization of a broadband alloptical ultrasound transducer-from optical and acoustical properties to imaging. Ultrasonics, Ferroelectrics and Frequency Control, IEEE Transactions on, 55(8):18671877, 2008. 
[5] Y Hou, S Ashkenazi, S-W Huang, and M O'Donnell. An integrated optoacoustic transducer combining etalon and black pdms structures. Ultrasonics, Ferroelectrics and Frequency Control, IEEE Transactions on, 55(12):27192725, 2008.

[6] E Biagi, S Cerbai, L Masotti, L Belsito, A Roncaglia, G Masetti, and N Speciale. Fiber optic broadband ultrasonic probe for virtual biopsy: Technological solutions. Journal of Sensors, 2010, 2010.

[7] C Sheaff and S Ashkenazi. An all-optical thin-film high-frequency ultrasound transducer. In International Ultrasonics Symposium, pages 1944-1947. IEEE, 2011.

[8] B-Y Hsieh, S-L Chen, T Ling, LJ Guo, and P-C Li. All-optical scanhead for ultrasound and photoacoustic imaging - imaging mode switching by dichroic filtering. Photoacoustics, 2(1):39-46, 2014.

[9] C Sheaff and S Ashkenazi. Polyimide-etalon all-optical ultrasound transducer for high frequency applications. In Proceedings SPIE BiOS, pages 89434M-89434M-8. International Society for Optics and Photonics, 2014.

[10] X Zou, N Wu, Y Tian, and X Wang. Broadband miniature fiber optic ultrasound generator. Optics express, 22(15):18119-18127, 2014.

[11] RJ Colchester, EZ Zhang, CA Mosse, PC Beard, I Papakonstantinou, and AE Desjardins. Broadband miniature optical ultrasound probe for high resolution vascular tissue imaging. Biomedical Optics Express, 6(4):15021511, 2015.

[12] HW Baac, JG Ok, A Maxwell, K-T Lee, Y-C Chen, AJ Hart, Z Xu, E Yoon, and LJ Guo. Carbon-nanotube optoacoustic lens for focused ultrasound generation and high-precision targeted therapy. Scientific reports, 2, 2012.

[13] A Karabutov, A Kaptilniy, AY Ivochkin, D Ksenofontov, and A Trofimov. Optoacoustic study of laser-induced near-critical states of thin aluminum films. Moscow University Physics Bulletin, 68(5):383-386, 2013.

[14] E Vannacci, L Belsito, F Mancarella, M Ferri, $\mathrm{G}$ Veronese, A Roncaglia, and E Biagi. Miniaturized fiber-optic ultrasound probes for endoscopic tissue analysis by micro-opto-mechanical technology. Biomedical microdevices, 16(3):415-426, 2014.

[15] SH Lee, M-a Park, JJ Yoh, H Song, EY Jang, YH Kim, S Kang, and YS Yoon. Reduced graphene oxide coated thin aluminum film as an optoacoustic transmitter for high pressure and high frequency ultrasound generation. Applied Physics Letters, 101(24):241909, 2012.

[16] RJ Colchester, CA Mosse, DS Bhachu, JC Bear, CJ Carmalt, IP Parkin, BE Treeby, I Papakonstantinou, and AE Desjardins. Laser-generated ultrasound with optical fibres using functionalised carbon nanotube composite coatings. Applied Physics Letters, 104(17):173502, 2014.

[17] O Balogun and TW Murray. Frequency domain photoacoustics using intensity-modulated laser sources. Nondestructive Testing and Evaluation, 26(3-4):335-351, 2011.

[18] B Lashkari and A Mandelis. Features of the frequencyand time-domain photoacoustic modalities. International Journal of Thermophysics, 34(8-9):1398-1404, 2013.
[19] C Özdemir. Inverse synthetic aperture radar imaging with MATLAB algorithms, volume 210. John Wiley \& Sons, 2012.

[20] T Misaridis and JA Jensen. Use of modulated excitation signals in medical ultrasound. part ii: design and performance for medical imaging applications. Ultrasonics, Ferroelectrics and Frequency Control, IEEE Transactions on, 52(2):192-207, 2005.

[21] S Telenkov, A Mandelis, B Lashkari, and M Forcht. Frequency-domain photothermoacoustics: Alternative imaging modality of biological tissues. Journal of Applied Physics, 105(10):102029, 2009.

[22] B Lashkari and A Mandelis. Photoacoustic radar imaging signal-to-noise ratio, contrast, and resolution enhancement using nonlinear chirp modulation. Optics letters, 35(10):1623-1625, 2010.

[23] S Kellnberger, NC Deliolanis, D Queirós, G Sergiadis, and $\mathrm{V}$ Ntziachristos. In vivo frequency domain optoacoustic tomography. Optics letters, 37(16):3423-3425, 2012.

[24] EZ Zhang and PC Beard. Characteristics of optimized fibre-optic ultrasound receivers for minimally invasive photoacoustic detection. In Proceedings SPIE BiOS, pages 932311-932311-9. International Society for Optics and Photonics, 2015.

[25] E Zhang, J Laufer, and P Beard. Backward-mode multiwavelength photoacoustic scanner using a planar fabryperot polymer film ultrasound sensor for high-resolution three-dimensional imaging of biological tissues. Applied optics, 47(4):561-577, 2008.

[26] EZ Zhang and PC Beard. A miniature all-optical photoacoustic imaging probe. In Proceedings SPIE BiOS, pages 78991F-78991F-6. International Society for Optics and Photonics, 2011.

[27] BE Treeby and BT Cox. k-wave: Matlab toolbox for the simulation and reconstruction of photoacoustic wave fields. Journal of biomedical optics, 15(2):021314021314-12, 2010.

[28] RJ Colchester, CA Mosse, DI Nikitichev, EZ Zhang, $S$ West, PC Beard, I Papakonstantinou, and AE Desjardins. Real-time needle guidance with photoacoustic and laser-generated ultrasound probes. In Proceedings SPIE BiOS, pages 932321-932321-5. International Society for Optics and Photonics, 2015.

[29] I Pelivanov, D Kopylova, N Podymova, and A Karabutov. Optoacoustic technique for thickness measurement of submicron metal coatings. Laser physics, 19(6):1350 1360, 2009.

[30] T Buma, M Spisar, and M O'Donnell. A high-frequency, 2-d array element using thermoelastic expansion in pdms. Ultrasonics, Ferroelectrics and Frequency Control, IEEE Transactions on, 50(9):1161-1176, 2003.

[31] A Karabutov, V Larichev, G Maksimov, I Pelivanov, and $\mathrm{N}$ Podymova. Relaxation dynamics of a broadband nanosecond acoustic pulse in a bubbly medium. Acoustical Physics, 52(5):582-588, 2006.

[32] A Karabutov, A Devichensky, A Ivochkin, M Lyamshev, I Pelivanov, U Rohadgi, V Solomatin, and M Subudhi. 
Laser ultrasonic diagnostics of residual stress. Ultrasonics, 48(6):631-635, 2008.

[33] T Ling, S-L Chen, and LJ Guo. High-sensitivity and wide-directivity ultrasound detection using high q polymer microring resonators. Applied physics letters, 98(20), 2011.

[34] Thorlabs. Thorlabs fibre specification, http://www.thorlabs.de/, 2015.

[35] A Gertsch, N Bush, D Birtill, and J Bamber. Toward characterizing the size of microscopic optical absorbers using optoacoustic emission spectroscopy. In Proceedings SPIE BiOS, pages $75641 \mathrm{M}-75641 \mathrm{M}-10$. International Society for Optics and Photonics, 2010.

[36] G Xu, JB Fowlkes, C Tao, X Liu, and X Wang. Photoacoustic spectrum analysis for microstructure characterization in biological tissue: Analytical model. Ultrasound in medicine \& biology, 2015. 